\title{
Atrial Ectopy as a Mediator of the Association Between Race and Atrial Fibrillation
}

\author{
Matthew A. Christensen, MD ${ }^{\star}$, , Kaylin T. Nguyen, MD, Phyllis K. Stein, PhD ${ }^{\star}$, Raymond B. \\ Fohtung, MD§, Elsayed Z. Soliman, MD\|, Thomas A. Dewland, MDף, Eric Vittinghoff, PhD, \\ MPH $^{\#}$, Bruce M. Psaty, MD, PhD ${ }^{\star *}$, Susan R. Heckbert, MD, $\mathrm{PhD}^{\dagger \dagger}$, and Gregory M. Marcus, \\ MD, MAS, FHRS*
}

*Electrophysiology Section, Division of Cardiology, Department of Medicine, University of California, San Francisco, California

tUniversity of Michigan Medical School, Ann Arbor, Michigan

‡Division of Cardiology, Department of Medicine, Washington University, St. Louis, Missouri

$\S$ Department of Medicine, Washington University, St. Louis, Missouri

IDepartment of Epidemiology and Prevention, Wake Forest University, Winston Salem, North Carolina

IKnight Cardiovascular Institute, Oregon Health and Science University, Portland, Oregon

\#Department of Epidemiology and Biostatistics, University of California, San Francisco, San Francisco, California

${ }^{*}$ Cardiovascular Health Research Unit, Departments of Medicine, Epidemiology, and Health Sciences, University of Washington, Seattle, Washington

${ }^{+\dagger}$ Group Health Research Institute, Group Health Cooperation, Seattle, Washington

\begin{abstract}
BACKGROUND—Blacks have a lower risk of AF despite having more AF risk factors, but the mechanism remains unknown. PAC burden is a recently identified risk factor for AF.

OBJECTIVES-To determine whether the burden of premature atrial contractions (PACs) explains racial differences in atrial fibrillation (AF) risk.
\end{abstract}

METHODS—PAC burden (number per hour) was assessed by 24-hour ambulatory ECG monitoring in a randomly selected subset of Cardiovascular Health Study participants. Participants

CORRESPONDING AUTHOR: Gregory M. Marcus, MD, MAS, 505 Parnassus Ave, M-1180B, Box 0124, San Francisco, CA 94143-0124, phone (415) 476-5706; fax (415) 353-9190, marcusg@ medicine.ucsf.edu. Drs. Christensen and Nguyen contributed equally to this manuscript as first authors.

Disclosures: Dr. Marcus has received research funding from Medtronic and Cardiogram, is a consultant for InCarda and Lifewatch and holds equity interest in InCarda; none of the other authors have any potential conflicts of interest.

Publisher's Disclaimer: This is a PDF file of an unedited manuscript that has been accepted for publication. As a service to our customers we are providing this early version of the manuscript. The manuscript will undergo copyediting, typesetting, and review of the resulting proof before it is published in its final citable form. Please note that during the production process errors may be discovered which could affect the content, and all legal disclaimers that apply to the journal pertain. 
were followed prospectively for the development of AF, diagnosed by study ECG and hospital admission records.

RESULTS-Among 938 participants (median age 73 years, 34\% Black, 58\% female), 206 (22\%) developed AF over a median follow-up of 11.0 years (IQR 6.1 to 13.4). After adjusting for age, sex, body mass index, coronary disease, congestive heart failure, diabetes, hypertension, alcohol consumption, smoking status, and study site, Black race was associated with a $42 \%$ lower risk of $\mathrm{AF}$ (HR $0.58,95 \%$ CI 0.40 to $0.85, \mathrm{p}=0.005$ ). The baseline PAC burden was 2.10 times ( $95 \% \mathrm{CI}$ 1.57 to $2.83, \mathrm{p}<0.001)$ higher in Whites than Blacks. There was no detectable difference in PVC burden by race. PAC burden mediated 19.5\% (95\% CI 6.3 to 52.5) of the adjusted association between race and $\mathrm{AF}$.

CONCLUSIONS-On average, Whites exhibited more PACs than Blacks, and this difference statistically explains a modest proportion of the differential risk of AF by race. The differential PAC burden, without differences in PVCs, by race suggests that identifiable common exposures or genetic influences might be important to atrial pathophysiology.

\section{Keywords}

Atrial fibrillation; Race; Premature atrial contractions; Atrial premature beats; Arrhythmia

\section{INTRODUCTION}

$\mathrm{AF}$ is the most common cardiac arrhythmia in the United States and contributes to significant morbidity and mortality. ${ }^{1,2}$ However, the mechanisms leading to AF remain incompletely understood. ${ }^{3}$ Recent studies have found that Blacks have a lower risk of AF compared to Whites, ${ }^{4-6}$ despite having a higher prevalence of AF risk factors such as hypertension and heart failure. ${ }^{7-9}$ Additionally, Blacks with a larger proportion of European ancestry have a higher risk of AF, suggesting a genetic component to the increased risk. ${ }^{10}$ The mechanism underlying the lower risk of $\mathrm{AF}$ in Blacks despite a higher prevalence of traditional AF risk factors remains to be fully understood. ${ }^{11}$ Elucidating the race-AF mechanism may provide new insights into the pathophysiology of AF.

Our group has previously demonstrated that more frequent premature atrial contractions (PACs) serve as a particularly accurate predictor of incident AF. ${ }^{12}$ PACs have also been shown to play an important role in AF pathophysiology, ${ }^{13}, 14$ and predict increased risk of stroke ${ }^{15}$ and death. ${ }^{16}$ Therefore, if the burden of PACs is different in Blacks and Whites, it is possible that PACs may be an important mediator of the paradoxical relationship between race and AF. Furthermore, since PAC burden is potentially modifiable by catheter ablation, examining how PACs mediate the race-AF relationship may guide targeted prevention strategies.

Therefore, we sought to test the hypothesis that Blacks have fewer PACs than Whites, and, if true, to determine whether the difference in PACs might explain in part the racial difference in incident AF. Specifically, we performed a mediation analysis in the random sample of participants in the Cardiovascular Health Study (CHS) who underwent 24-hour ECG ("Holter") monitoring. To assess whether PACs specifically (as opposed to ectopy in 
general) were important, we analyzed premature ventricular contraction (PVC) frequencies as a comparator.

\section{METHODS STUDY DESIGN}

CHS is a prospective community-based cohort of older adults that has a long duration of follow-up, rigorous measurements, and adjudicated event ascertainment which has been described in detail previously. ${ }^{17}$ Briefly, during 1989-90 an initial group of 5,201 adults (94.7\% White, $4.7 \%$ Black, $0.6 \%$ other race) age $\geq 65$ years were recruited from a random sample of Medicare beneficiaries at 4 U.S. sites - Forsyth County, NC; Sacramento County, CA; Washington County, MD; Pittsburgh, PA — and an additional 678 Blacks (and nine of other races) were recruited from 1992-93. The institutional review board at all clinical centers and the coordinating center approved the study and all participants provided informed consent. Participants underwent a comprehensive baseline examination including standardized questionnaires, repeated blood pressure measurements, physical exam, and 12lead ECG. CHS participants were followed by alternating semiannual phone calls and clinic visits until 1999 and by phone calls every 6 months thereafter. Certification to use deidentified CHS data was obtained from the University of California, San Francisco Institutional Review Board.

\section{STUDY COHORT}

Our analysis was restricted to a subset of 1,250 participants that were randomly assigned to 24-hour ambulatory ECG (Holter) monitoring during the 1994-95 examination. Compared to Holter data obtained from the initial group recruited during 1989-90, this second subset included substantially more Blacks ( $34 \%$ vs. $4 \%$ ). Because not all covariates were ascertained at the 1994-95 examination, covariates collected at the 1992-93 examination were carried forward. The 1994-95 examination was used as the origin time for prospective analyses on the risk of AF.

\section{PAC AND PVC ASSESSMENT}

Holter data were analyzed at the Washington University School of Medicine Heart Rate Variability Laboratory using a MARS 8000 Holter scanner (GE Healthcare, Milwaukee, Wisconsin) and manually reviewed to ensure accuracy. Participants with atrial pacing, wandering atrial pacemaker, or poor-quality Holter data were excluded. The frequency (events/hour) of PACs and PVCs was calculated as the total number of PACs divided by the duration of monitoring in hours.

\section{COVARIATE ASSESSMENT AND DEFINITION}

Covariates were collected at the 1992-93 (year 5) study examination, the study exam that collected the most relevant data closest to and previous to the year 7 Holter, and carried forward. Self-reported race was dichotomized as Black versus White and those who reported other races or had missing race status were excluded from analyses. Height was assessed barefoot with a wall-mounted stadiometer to the nearest $0.1 \mathrm{~cm}$. Weight was quantified using a balance beam scale to the nearest $0.1 \mathrm{~kg}$. Body mass index (BMI) was calculated by 
dividing weight in kilograms by height in meters squared. Coronary heart disease (CHD) and congestive heart failure (CHF) were identified at initial examination (by self-report, examination or review of medical records $)^{18}$ and updated for incident disease identified by annual study visit and review of medical records (discharge diagnosis codes supplemented by Medicare claims data) up through the 1992-93 (year 5) examination. Diabetes was present if the participant had a fasting glucose concentration $\geq 126 \mathrm{mmol} / \mathrm{L}$ or used an oral hypoglycemic agent or insulin. Hypertension (HTN) was defined as systolic blood pressure $\geq 140 \mathrm{~mm} \mathrm{Hg}$, diastolic blood pressure $\geq 95 \mathrm{~mm} \mathrm{Hg}$, or as the use of anti-hypertensive medication with a history of physician-diagnosed hypertension. Alcohol use was selfreported as average number of drinks per week and dichotomized as consumption of $\geq 1$ drinks per week versus less. Smoking status was identified by self-report as never (smoked < 100 cigarettes ever), past, and current. Resting 12-lead ECGs were recorded using MAC PC ECG Machines (Marquette Electronics) and processed using the GE Marquette 12-SL program. The PR interval measured from the 12-lead ECG obtained during the 1994-95 visit was used. Participants with missing data for any covariates (at the 1992-93 examination) were excluded.

\section{AF ASCERTAINMENT}

AF was ascertained from the serial 12-lead ECGs conducted at each annual clinic visit through 1999 and medical records (discharge diagnosis codes supplemented by Medicare claims data) for all encounters after enrollment. Incident AF was defined as the time that AF by any of these measures was first identified. Those with prevalent AF at the time of Holter recording or before the 1994-94 examination were excluded. Follow-up ended at the time of AF diagnosis, time of death, or at the end of the study period.

\section{STATISTICAL ANALYSES}

Normally distributed continuous variables are presented as means $\pm \mathrm{SD}$, and non-normally distributed variables are presented as medians and interquartile ranges (IQRs). Variables were compared across race using t-tests, Wilcoxon rank-sum tests, Chi-square tests, or Fisher's exact tests as appropriate. Linear regression was used to assess the crude and adjusted association between race and frequency of PACs and PVCs. To meet the requirement of normally distributed outcomes in linear models, both PAC and PVC frequency were transformed using $\log$ base $2\left(\log _{2}\right)$ with addition of a 0.01 constant to retain zero values, and normality was subjectively assessed by comparing histograms and Q-Q plots. For all linear regression models, component-plus-residual plots were used to assess model linearity, Shapiro-Wilks tests and Q-Q plots were used to assess normality of the residuals, residual versus fitted plots were used to assess constant variance, and dfbeta statistics were used to identify outliers and assess their effect. Of all predictors, only BMI demonstrated non-linearity which was remedied by a BMI-squared term, but results were not meaningfully different. Similarly, there were a small number of influential points in several models, but exclusion did not change our findings.

$\log _{2}$-transformed PAC frequency was associated with race so it was carried forward in a mediation analysis. Cox proportional hazards models were used to assess the crude and adjusted association between Black race and incident AF before and after inclusion of $\log _{2}$ - 
transformed PAC frequency in the model. The proportional hazards assumption was assessed using scaled Schoenfeld residuals and the assumption of linearity was assessed with inclusion of cubic-spline terms for continuous predictors. The assumptions of proportional hazards and linearity were met in all Cox models. The "percent treatment effect" methodology was used to assess the degree of mediation by PACs before and after adjustment for potential confounders. ${ }^{19}$ Confidence intervals (CI) for mediation statistics were obtained using bootstrap resampling with 500 repetitions. Covariates in adjusted models included age, sex, BMI, CHD, CHF, diabetes, HTN, alcohol consumption, smoking status, and study site. A sensitivity analysis was conducted with additional adjustment for PR interval.

All analyses were performed using Stata 14 (StataCorp LP, College Station, TX). A 2-tailed $\mathrm{p}<0.05$ was considered statistically significant.

\section{RESULTS \\ PARTICIPANTS}

Compared with other participants in CHS, those who underwent Holter monitoring tended to be slightly younger, had a higher baseline BMI, consumed less alcohol, and had a lower prevalence of heart disease (CHD and CHF) (Supplemental Table 1). Among the 1,250 participants (65.5\% White, 33.8\% Black, $0.6 \%$ others) who underwent Holter monitoring, a total of $312(25 \%)$ were excluded from further analysis ( 8 neither Black nor White race; 107 with prevalent AF up to the time of Holter monitoring; 129 for atrial pacing, wandering atrial pacemaker, or poor-quality Holter data; 68 for missing any of the other covariates). Among the remaining cohort of 938 participants, 318 (34\%) were Black. Baseline characteristics of the included participants are shown in Table 1. Black participants were younger on average, more likely to be a current smoker, had a greater mean BMI, and had a higher prevalence of HTN and diabetes.

\section{ECTOPY AND RACE}

Over a median Holter monitoring duration of 23.8 hours (IQR 23.0 to 24.3), the median PAC burden was 2.5 events/hour (IQR 0.7 to 11.9), and the median PVC burden was 1.2 events/ hour (IQR 0.1 to 10.6). On average, Whites exhibited a significantly higher burden of PACs than Blacks, without any statistically significant differences in PVCs (Figure 1). In unadjusted analysis, Whites had a PAC burden that was 2.10 times (95\% CI 1.57 to 2.83, $\mathrm{p}<0.001)$ higher than that in Blacks. After adjusting for age, sex, BMI, CHD, CHF, diabetes, HTN, alcohol consumption, smoking status, and study site, Whites exhibited 1.59 (95\% CI 1.14 to 2.20, $\mathrm{p}=0.006$ ) times as many PACs as Blacks. The burden of PVCs was also not significantly different after multivariable adjustment.

\section{MEDIATION ANALYSIS}

Over a median follow-up of 11.0 years (IQR 6.1 to 13.4), 206 (22\%) participants developed AF. The crude incidence rate of AF was 1.5 per 100 person-years among Blacks and 2.7 per 100 person-years among Whites. After adjustment for age, sex, BMI, CHD, CHF, diabetes, HTN, alcohol consumption, smoking status, and study site, Black race was associated with a 
$42 \%$ lower risk of AF (HR $0.58,95 \%$ CI 0.40 to $0.85, \mathrm{p}=0.005$ ) (Figure 2). Upon addition of $\mathrm{PAC}$ burden to the model, the protective effect of Black race against $\mathrm{AF}$ was diminished to a $34 \%$ lower risk of incident AF (HR 0.66, 95\% CI 0.45 to $0.95, \mathrm{p}=0.026$ ). The PAC burden accounted for $19.5 \%$ (bootstrap 95\% CI 6.3 to 52.5) of the association of Black race with reduced risk of AF. These results were not meaningfully different in a sensitivity analysis that additionally controlled for the PR interval.

\section{DISCUSSION}

In a large, community-based sample of older Black and White adults who underwent 24hour Holter monitoring, Blacks had fewer PACs than Whites, without evidence of significant racial difference in PVC burden. Consistent with previous reports, Blacks had a lower rate of incident AF compared to Whites before and after controlling for other established risk factors. Attenuation of the race-AF association after controlling for baseline PAC burden indicates that approximately $20 \%$ of the reduced risk of AF among Blacks may be attributable to racial differences in atrial ectopy.

The race-AF association has been established in several settings, ${ }^{5,6,11,20}$ and Whites appear to be at a heightened risk compared to multiple other races despite having fewer AF risk factors. ${ }^{4}$ Recent evidence suggests that socio-economic-status does not explain these differences, ${ }^{21}$ but increasing European ancestry is an independent risk factor for AF in Blacks, ${ }^{10}$ suggesting instead that genetically mediated racial differences play a role. However, the underlying mechanisms explaining the racial differences in risk of AF are largely unknown. Identification of mediators of this naturally occurring difference may lead to novel insights into AF pathogenesis.

Higher baseline levels of several inflammatory markers have been shown to mediate approximately $40 \%$ of the heightened risk of AF among Whites, ${ }^{22}$ but it is difficult to directly modify the degree of baseline inflammation. In addition, the mechanism by which inflammation may lead to AF remains largely unknown - for example, inflammation itself may induce more ectopy. Atrial ectopy is important for initiating episodes of $\mathrm{AF},{ }^{13}$ is an independent risk factor for $\mathrm{AF}$ that is as predictive as an optimized combination of traditional AF risk factors, ${ }^{12}$ and is potentially modifiable by catheter ablation or antiarrhythmic drug therapy. The emerging importance of PACs motivated our hypothesis that racial differences in PAC burden might explain the race-AF association.

Our finding that Blacks had fewer PACs but a similar number of PVCs compared to Whites suggests that these racial differences in ectopy are specific to the atria. The significant attenuation of the race-AF association by PAC burden even after adjustment for traditional AF risk factors supports an underlying pathophysiology specific to the atria that can be identified well before AF itself develops. Of note, the PACs we observed statistically explained only approximately $20 \%$ of the relationship between race and AF (although the upper end of the $95 \%$ confidence interval suggests that PACs could explain up to a half of the race-AF relationship). Therefore, there may be other potential mechanisms at play, such as additional interactions with certain genetic variants or environmental exposures, or perhaps 24 hour Holter monitoring did not sufficiently capture the true differential rates of 
ectopy over a lifetime. Regardless of specific mechanisms, our results support inclusion of PACs in AF risk stratification and suggest that evaluation of baseline PAC burden may aid in identifying patient regardless of race who have an elevated risk of AF. Baseline PAC evaluation might be studied in the future as a means to identify those particularly amenable to various therapies.

More broadly, these findings suggest that ectopy is not necessarily idiosyncratic to a given individual and does not simply occur by chance alone. These observations, that selfidentified groups known to share a common ancestry exhibited differential amounts of atrial ectopy, suggest that there is either a genetic origin or some common exposure that is important. This then suggests that there is some modifiable risk factor(s) might be identified, or, if genetic, that the fundamental mechanisms responsible for AF might be elucidated and then directly addressed.

\section{STUDY LIMITATIONS}

Several potential limitations of this analysis should be considered. First, residual confounding remains a possibility. For example, some covariates not accounted for in our multivariate models such as obstructive sleep apnea, chronic kidney disease and height itself (apart from BMI), could have accounted for some of the differences observed. Second, differential treatment by race in healthcare settings is a valid concern and could lead to a falsely reduced incidence of AF among Blacks. However, since Blacks and Whites in CHS were randomly selected to undergo Holter monitoring, differential ascertainment of PAC (and PVC) burden by race is unlikely. Like most other studies evaluating race and AF, race was based upon self-report, although misclassification would likely bias our results towards the null hypothesis. Because AF was ascertained using ECGs and discharge diagnoses, it is possible that some asymptomatic paroxysmal AF was not detected. However, this would likely reduce our power and should not result in false positive relationships. It is possible that Blacks have AF that less often comes to medical attention or, based on recent evidence that certain patient characteristics are associated with paroxysmal versus persistent $\mathrm{AF},{ }^{23}$ that Blacks more often experience asymptomatic paroxysmal AF that would more likely go undetected. However, a recent study among patients with pacemakers suggests that, even among patients that are continuously monitored, Blacks exhibit less overall $\mathrm{AF}^{24}$ Finally, since this is an observational study we cannot make any conclusions regarding causation. For example, it remains possible that PACs are an epi-phenomenon shared with AF, reflecting a common atrial myopathy as the underlying source. However, this would not negate the fact that PACs preceding AF may still serve as a useful predictor.

\section{CONCLUSION}

In a community-based sample of older adults, we found that Blacks have significantly fewer PACs at baseline, explaining approximately $20 \%$ of the reduced risk of incident AF among Blacks compared to Whites. This mediation persisted after controlling for other AF risk factors, suggesting that PACs play a key role in explaining racial differences in AF onset. Given no difference in PVC burden, these data suggest that racial differences may be uniquely relevant to the atria. These observations might inspire future investigations seeking 
to explain how differential racial exposures or genetic influences might affect the propensity for ectopy and pathological atrial characteristics years before AF develops.

\section{Supplementary Material}

Refer to Web version on PubMed Central for supplementary material.

\section{Acknowledgments}

FUNDING:

This work was supported by the Sarnoff Cardiovascular Research Foundation and the Division of Cardiology, Department of Medicine, UCSF under Dr. Marcus, National Institute on Minority Health and Health Disparities [R25MD006832], National Institute on Aging (NIA) [R01AG023629]. The Cardiovascular Health Study was supported by National Heart, Lung, and Blood Institute (NHLBI), with additional contribution from the National Institute of Neurological Disorders and Stroke (NINDS) [HHSN268201200036C, HHSN268200800007C, N01HC55222, N01HC85079, N01HC85080, N01HC85081, N01HC85082, N01HC85083, N01HC85086, and grants U01HL080295 and U01HL130114].

Mr. Christensen was a research fellow supported by the Sarnoff Cardiovascular Research Foundation and the Division of Cardiology, Department of Medicine, UCSF under Dr. Marcus. Ms. Nguyen was a research fellow supported by R25MD006832 from the National Institute on Minority Health and Health Disparities. The Cardiovascular Health Study was supported by contracts HHSN268201200036C, HHSN268200800007C, N01HC55222, N01HC85079, N01HC85080, N01HC85081, N01HC85082, N01HC85083, N01HC85086, and grants U01HL080295 and U01HL130114 from the National Heart, Lung, and Blood Institute (NHLBI), with additional contribution from the National Institute of Neurological Disorders and Stroke (NINDS). Additional support was provided by R01AG023629 from the National Institute on Aging (NIA). A full list of principal CHS investigators and institutions can be found at CHS-NHLBI.org. The content is solely the responsibility of the authors and does not necessarily represent the official views of the National Institutes of Health.

\section{References}

1. Benjamin EJ, Wolf PA, D’Agostino RB, Silbershatz H, Kannel WB, Levy D. Impact of atrial fibrillation on the risk of death: the Framingham Heart Study. Circulation [Internet]. 1998; 98:946952. Available from: http://www.ncbi.nlm.nih.gov/pubmed/9737513.

2. Go AS, Mozaffarian D, Roger VL, et al. Heart disease and stroke statistics--2014 update: a report from the American Heart Association. Circulation [Internet]. 2014; 129:e28-e292. Available from: http://www.ncbi.nlm.nih.gov/pubmed/24352519.

3. January CT, Wann LS, Alpert JS, et al. 2014 AHA/ACC/HRS Guideline for the Management of Patients With Atrial Fibrillation: Executive Summary: A Report of the American College of Cardiology/American Heart Association Task Force on Practice Guidelines and the Heart Rhythm Society. J Am Coll Cardiol [Internet]. Journal of the American College of Cardiology. 2014; 64:2246-2280. [cited 2014 Jul 9]. Available from: http://content.onlinejacc.org/article.aspx? articleid $=1854230$.

4. Dewland TA, Olgin JE, Vittinghoff E, Marcus GM. Incident atrial fibrillation among Asians, Hispanics, Blacks, and Whites. Circulation [Internet] United States. 2013; 128:2470-2477. Available from: http://www.ncbi.nlm.nih.gov/pubmed/24103419.

5. Alonso A, Agarwal SK, Soliman EZ, Ambrose M, Chamberlain AM, Prineas RJ, Folsom AR. Incidence of atrial fibrillation in Whites and African-Americans: the Atherosclerosis Risk in Communities (ARIC) study. Am Heart J [Internet]. 2009; 158:111-117. Available from: http:// www.ncbi.nlm.nih.gov/pubmed/19540400.

6. Marcus GM, Olgin JE, Whooley M, Vittinghoff E, Stone KL, Mehra R, Hulley SB, Schiller NB. Racial Differences in Atrial Fibrillation Prevalence and Left Atrial Size. Am J Med [Internet] Elsevier Inc. 2010; 123:375e1-375.e7. Available from: http://dx.doi.org/10.1016/j.amjmed. 2009.05.019. 
7. Hertz RP, Unger AN, Cornell JA, Saunders E. Racial disparities in hypertension prevalence, awareness, and management. Arch Intern Med [Internet]. 2005; 165:2098-2104. Available from: http://archinte.jamanetwork.com/article. aspx?articleid=486726.

8. Bahrami H, Kronmal R, Bluemke DA, Olson J, Shea S, Liu K, Burke GL, Lima JAC. Differences in the incidence of congestive heart failure by ethnicity: the Multi-Ethnic Study of Atherosclerosis. Arch Intern Med [Internet]. 2008; 168:2138-2145. Available from: http://dx.doi.org/10.1001/ archinte.168.19.2138.

9. Kalogeropoulos A, Georgiopoulou V, Kritchevsky SB, et al. Epidemiology of Incident Heart Failure in a Contemporary Elderly Cohort The Health, Aging, and Body Composition Study. Arch Intern Med [Internet]. 2009; 169:708-715. Available from: http://www.pubmedcentral.nih.gov/ articlerender.fcgi?artid=2756793\&tool=pmcentrez\&rendertype $=$ abstract.

10. Marcus GM, Alonso A, Peralta CA, et al. European ancestry as a risk factor for atrial fibrillation in African Americans. Circulation. 2010; 122:2009-2015. [PubMed: 21098467]

11. Jensen PN, Thacker EL, Dublin S, Psaty BM, Heckbert SR. Racial differences in the incidence of and risk factors for atrial fibrillation in older adults: The Cardiovascular Health Study. J Am Geriatr Soc. 2013; 61:276-280. [PubMed: 23320758]

12. Dewland TA, Vittinghoff E, Mandyam MC, Heckbert SR, Siscovick DS, Stein PK, Psaty BM, Sotoodehnia N, Gottdiener JS, Marcus GM. Atrial ectopy as a predictor of incident atrial fibrillation: a cohort study. Ann Intern Med [Internet] American College of Physicians. 2013; 159:721-728. [cited 2016 Jun 29]. Available from: http://annals.org/article.aspx? doi=10.7326/0003-4819-159-11-201312030-00004.

13. Haïssaguerre M, Jaïs P, Shah DC, Takahashi A, Hocini M, Quiniou G, Garrigue S, Le Mouroux A, Le Métayer P, Clémenty J. Spontaneous initiation of atrial fibrillation by ectopic beats originating in the pulmonary veins. N Engl J Med. 1998; 339:659-666. [PubMed: 9725923]

14. Andrade J, Khairy P, Dobrev D, Nattel S. The Clinical Profile and Pathophysiology of Atrial Fibrillation: Relationships Among Clinical Features, Epidemiology, and Mechanisms. Circ Res [Internet] United States. 2014; 114:1453-1468. Available from: http:// circres.ahajournals.org/cgi/doi/10.1161/CIRCRESAHA.114.303211.

15. Larsen B, Kumarathurai P, Falkenberg J, Nielsen OW, Sajadieh A. Excessive Atrial Ectopy and Short Atrial Runs Increase the Risk of Stroke beyond Incident Atrial Fibrillation. J Am Coll Cardiol. 2015; 66:232-241. [PubMed: 26184616]

16. Qureshi W, Shah AJ, Salahuddin T, Soliman EZ. Long-term mortality risk in individuals with atrial or ventricular premature complexes (results from the Third National Health and Nutrition Examination Survey). Am J Cardiol [Internet]. 2014; 114:59-64. Available from: http:// www.ncbi.nlm.nih.gov/pubmed/24819898.

17. Fried LP, Borhani NO, Enright P, Furberg CD, Gardin JM, Kronmal RA, Kuller LH, Manolio TA, Mittelmark MB, Newman A. The Cardiovascular Health Study: design and rationale. Ann Epidemiol [Internet]. 1991; 1:263-276. Available from: http://www.ncbi.nlm.nih.gov/pubmed/ 1669507.

18. Psaty BM, Kuller LH, Bild D, Burke GL, Kittner SJ, Mittelmark M, Price TR, Rautaharju PM, Robbins J. Methods of assessing prevalent cardiovascular disease in the Cardiovascular Health Study. Ann Epidemiol. 1995; 5:270-277. [PubMed: 8520708]

19. Lin D, Fleming T, De Gruttola V. Estimating the proportion of treatment effect explained by a surrogate marker. Stat Med. 1997; 16:1515-1527. [PubMed: 9249922]

20. Go AS, Hylek EM, Phillips KA, Chang Y, Henault LE, Selby JV, Singer DE. Prevalence of diagnosed atrial fibrillation in adults: national implications for rhythm management and stroke prevention: the AnTicoagulation and Risk Factors in Atrial Fibrillation (ATRIA) Study. JAMA. 2001; 285:2370-2375. [PubMed: 11343485]

21. Misialek, JR., Rose, KM., Everson-Rose, SA., Soliman, EZ., Clark, CJ., Lopez, FL., Alonso, A. J Am Heart Assoc [Internet]. Vol. 3. Lippincott Williams \& Wilkins; 2014. Socioeconomic Status and the Incidence of Atrial Fibrillation in Whites and Blacks: The Atherosclerosis Risk in Communities (ARIC) Study; p. e001159-e001159. [cited 2016 Jun 28]Available from: http:// jaha.ahajournals.org/cgi/doi/10.1161/JAHA.114.001159

22. Dewland TA, Vittinghoff E, Harris TB, Magnani JW, Liu Y, Hsu F-C, Satterfield S, Wassel C, Marcus GM. Inflammation as a Mediator of the Association Between Race and Atrial Fibrillation. 
J AM Coll Cardiol EP [Internet]. 2015; 1:248-255. [cited 2016 Jun 28]; Available from: http:// linkinghub.elsevier.com/retrieve/pii/S2405500X15001899.

23. Sandhu RK, Conen D, Tedrow UB, Fitzgerald KC, Pradhan AD, Ridker PM, Glynn RJ, Albert CM. Predisposing factors associated with development of persistent compared with paroxysmal atrial fibrillation. J Am Heart Assoc [Internet]. 2014; 3:e000916. Available from: http:// www.ncbi.nlm.nih.gov/pubmed/24786144.

24. Kamel, H., Kleindorfer, DO., Bhave, PD., Cushman, M., Levitan, EB., Howard, G., Soliman, EZ. Rates of Atrial Fibrillation in Black Versus White Patients With Pacemakers; J Am Heart Assoc [Internet]. 2016. p. 5Available from: http://www.ncbi.nlm.nih.gov/pubmed/26873685 


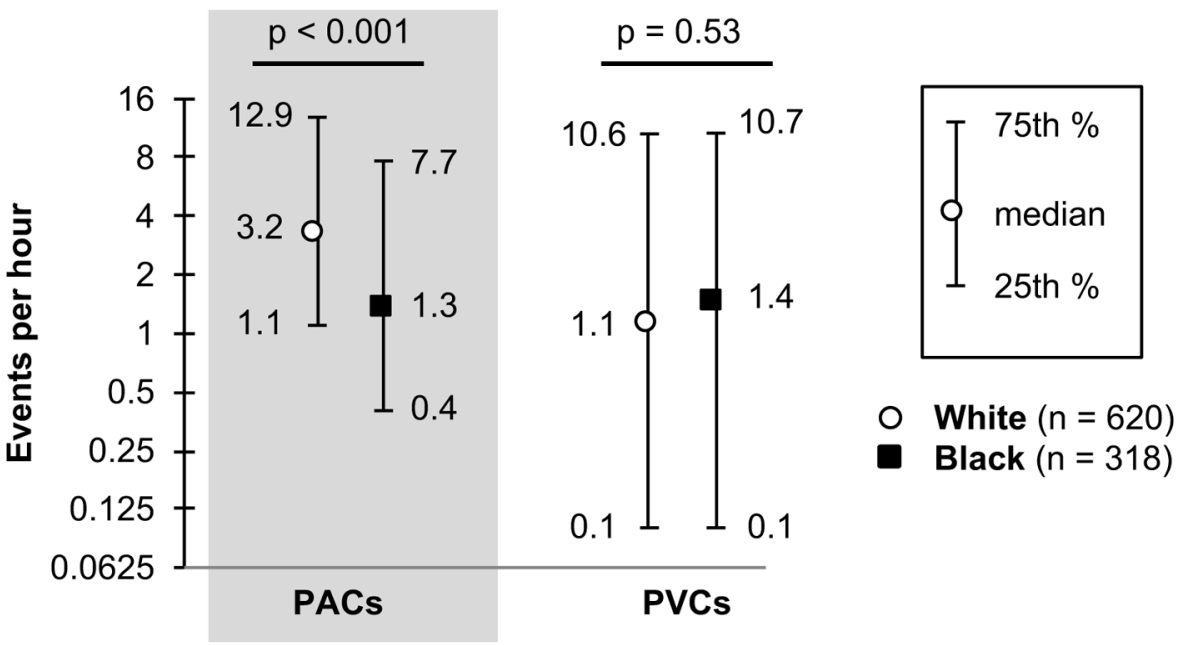

FIGURE 1. PAC and PVC Burden by Race

White circles and black squares represent median event rates among Whites and Blacks, respectively, plotted on a $\log _{2}$ scale. Bars represent interquartile ranges $\left(25^{\text {th }}\right.$ percentile to $75^{\text {th }}$ percentile). P-values were obtained from bivariate linear regression models. Abbreviations: $\mathrm{PAC}=$ premature atrial contraction $\mathrm{PVC}=$ premature ventricular contraction. 


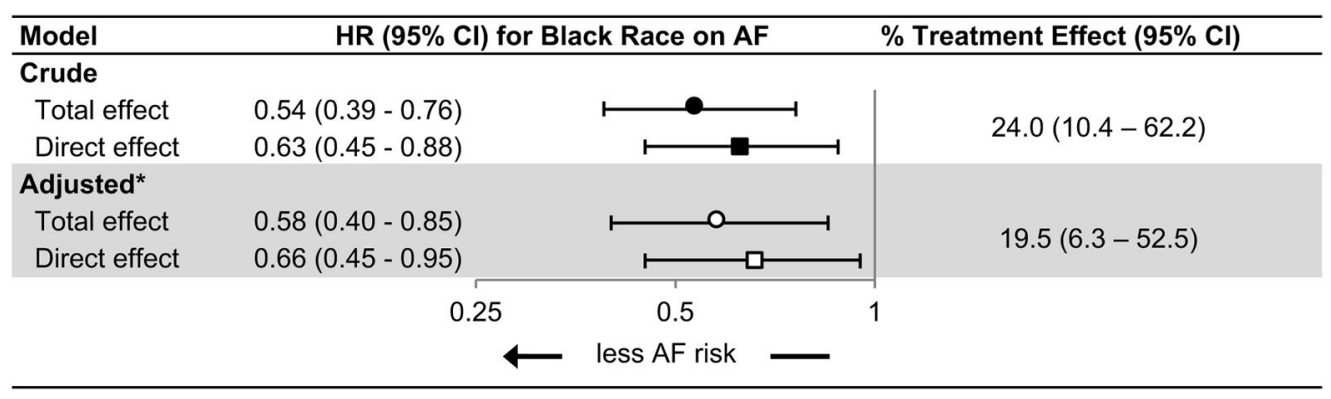

\section{FIGURE 2. Mediation of Race-AF Relationship by PAC Burden}

Circles and squares correspond to hazard ratios for the effect of Black race, compared to White, on AF. Direct effect is the hazard for AF associated with race after adjustment for baseline PAC burden. \% Treatment Effect corresponds to the degree of mediation by baseline PAC burden. Bars represent 95\% CIs. *Adjusted for age, sex, body mass index, coronary disease, congestive heart failure, diabetes, hypertension, alcohol consumption, smoking status, and study site.

Abbreviations: $\mathrm{AF}=$ atrial fibrillation; $\mathrm{CI}=$ confidence interval; $\mathrm{HR}=$ hazard ratio; $\mathrm{PAC}=$ premature atrial contraction. 
TABLE 1

Characteristics of Participants by Race $(\mathrm{N}=938)$

\begin{tabular}{lccc}
\hline Characteristic & White (N= 620) & Black (N = 318) & P-Value \\
\hline Age, median (IQR), years & $73(71-77)$ & $71(68-75)$ & $<0.001$ \\
\hline Female sex, n (\%) & $345(56 \%)$ & $198(62 \%)$ & 0.052 \\
\hline BMI, mean \pm SD, kg/m² & $26.9 \pm 4.2$ & $28.6 \pm 5.1$ & 0.003 \\
\hline Alcohol consumption $\geq 1$ drink/week, n (\%) & $176(28 \%)$ & $75(24 \%)$ & 0.12 \\
\hline Smoking status, n (\%) & $273(44 \%)$ & $149(47 \%)$ & \\
Never & $302(49 \%)$ & $124(39 \%)$ & $<0.001$ \\
Former & $45(7 \%)$ & $45(14 \%)$ & \\
Current & $121(20 \%)$ & $54(17 \%)$ & 0.35 \\
\hline CHD, n (\%) & $21(3 \%)$ & $17(5 \%)$ & 0.15 \\
\hline CHF, n (\%) & $93(15 \%)$ & $67(21 \%)$ & 0.019 \\
\hline Diabetes, n (\%) & $255(41 \%)$ & $196(62 \%)$ & $<0.001$ \\
\hline HTN, n (\%) & & & \\
\hline
\end{tabular}

T-tests were used for normally distributed continuous variables (BMI); Wilcoxon Rank-Sum tests were used for non-normally distributed continuous variables (age); Chi-sqaure tests were used for categorical variables (all others).

Abbreviations: $\mathrm{BMI}=$ body mass index $\mathrm{CHD}=$ coronary heart disease $; \mathrm{CHF}=$ congestive heart failure; $\mathrm{HTN}=$ hypertension; IQR $=$ interquartile range; $\mathrm{SD}=$ standard deviation. 